\title{
Asymptotic error expansion of wavelet approximations of smooth functions II
}

\author{
Wim Sweldens ${ }^{1,2, \star}$ and Robert Piessens ${ }^{2}$ \\ 1 University of South Carolina, Department of Mathematics, Columbia, SC 29208, USA \\ 2 Katholieke Universiteit Leuven, Department of Computer Science, Celestijnenlaan 200A, B-3001 Leuven, \\ Belgium \\ e-mail: sweldens@math.scarolina.edu
}

Received May 3, 1993 / Revised version received January 31, 1994

Summary. We generalize earlier results concerning an asymptotic error expansion of wavelet approximations. The properties of the monowavelets, which are the building blocks for the error expansion, are studied in more detail, and connections between spline wavelets and Euler and Bernoulli polynomials are pointed out. The expansion is used to compare the error for different wavelet families. We prove that the leading terms of the expansion only depend on the multiresolution subspaces $V_{j}$ and not on how the complementary subspaces $W_{j}$ are chosen. Consequently, for a fixed set of subspaces $V_{j}$, the leading terms do not depend on the fact whether the wavelets are orthogonal or not. We also show that Daubechies' orthogonal wavelets need, in general, one level more than spline wavelets to obtain an approximation with a prescribed accuracy. These results are illustrated with numerical examples.

Mathematics Subject Classification (1991): 41A30, 65D32, 42C05

\section{Notation}

A measurable function $f(x)$ belongs to the space $\mathrm{L}^{p}(\mathbb{R}), 1 \leq p<\infty$, if

$$
\|f(x)\|_{p}=\left(\int_{-\infty}^{+\infty}|f(x)|^{p} d x\right)^{1 / p}<\infty,
$$

and to $L^{\infty}(\mathbb{R})$ if

$$
\|f(x)\|_{\infty}=\sup _{x \in \mathrm{R}}|f(x)|<\infty .
$$

The inner product of two functions $f(x)$ and $g(x)$ of the Hilbert space $\mathrm{L}^{2}(\mathbb{R})$ is defined as

$$
\langle f, g\rangle=\int_{-\infty}^{+\infty} f(x) g(x) d x .
$$

\footnotetext{
* This author is partially supported by NSF EPSCoR Grant EHR 9108772 and ONR Grant N00014-90J-1343. He is also Research Assistant of the National Fund of Scientific Research Belgium Correspondence to: Wim Sweldens
} 
The Fourier transform of a function $f(x)$ is given by

$$
\widehat{f}(\omega)=\int_{-\infty}^{+\infty} f(x) \mathrm{e}^{-\mathrm{i} \omega x} d x .
$$

A countable set $\left\{f_{n}\right\}$ of a Hilbert space is a Riesz basis if every element $f$ of the space can be written uniquely as $f=\sum_{n} c_{n} f_{n}$, and positive constants $A$ and $B$ exist such that

$$
A\|f\|^{2} \leq \sum_{n}\left|c_{n}\right|^{2} \leq B\|f\|^{2} .
$$

The space $\mathscr{D}^{m}$ is defined a the space of bounded functions that decay faster than an inverse polynomial,

$$
\mathscr{D}^{m}=\left\{f(x)|| f(x) \mid \leq C(1+|x|)^{-(m+1+\epsilon)}, \epsilon>0\right\} .
$$

For a function of this space, its first $m+1$ moments are finite.

\section{Introduction}

We first briefly review wavelets and multiresolution analysis. For more detailed treatments, one can consult $[4,9,18,23,24]$.

\subsection{Multiresolution analysis}

A multiresolution analysis of $\mathrm{L}^{2}(\mathbb{R})$ is defined as a set of closed subspaces $V_{j}$ with $j \in \mathbb{Z}$ that exhibit the following properties:

1. $V_{j} \subset V_{j+1}$,

2. $v(x) \in V_{j} \Leftrightarrow v(2 x) \in V_{j+1}$ and $v(x) \in V_{0} \Leftrightarrow v(x+1) \in V_{0}$,

3. $\bigcup_{j=-\infty}^{+\infty} V_{j}$ is dense in $\mathrm{L}^{2}(\mathbb{R})$ and $\bigcap_{j=-\infty}^{+\infty} V_{j}=\{\mathbf{0}\}$,

4. A scaling function $\varphi(x) \in V_{0}$ exists such that the set $\{\varphi(x-l) \mid l \in \mathbb{Z}\}$ is a Riesz basis of $V_{0}$.

As a result a sequence $\left\{h_{k} \mid k \in \mathbb{Z}\right\}$, exists such that the scaling function satisfies a refinement equation

$$
\varphi(x)=2 \sum_{k} h_{k} \varphi(2 x-k) .
$$

The set of functions $\left\{\varphi_{j, l} \mid l \in \mathbb{Z}\right\}$ with $\varphi_{j, l}(x)=\sqrt{ } 2^{j} \varphi\left(2^{j} x-l\right)$, is a Riesz basis of $V_{j}$. Let $W_{j}$ be a complementary space of $V_{j}$ in $V_{j+1}$, such that $V_{j+1}=V_{j} \oplus W_{j}$. Consequently

$$
\bigoplus_{j=-\infty}^{+\infty} W_{j}=\mathrm{L}^{2}(\mathbb{R})
$$

Note that $W_{j}$ is not unique.

A function $\psi(x)$ is a wavelet if the set of functions $\{\psi(x-l) \mid l \in \mathbb{Z}\}$ is a Riesz basis of $W_{0}$. Since the wavelet is also an element of $V_{0}$, a sequence $\left\{g_{k} \mid k \in \mathbb{Z}\right\}$ exists such that 


$$
\psi(x)=2 \sum_{k} g_{k} \varphi(2 x-k)
$$

The set of wavelet functions $\left\{\psi_{j, l}(x) \mid l, j \in \mathbb{Z}\right\}$ is a Riesz basis of $\mathrm{L}^{2}(\mathbb{R})$.

The projection operator onto $V_{j}$ (resp. $W_{j}$ ) that corresponds to this splitting of $\mathrm{L}^{2}$ is denoted by $\mathscr{P}_{j}$ (resp. $\mathscr{Q}_{j}$ ). It can be written with the use of a dual scaling function $\widetilde{\varphi}($ resp. wavelet $\widetilde{\psi}$ ) as

$$
\mathscr{P}_{j} f(x)=\sum_{l}\left\langle f, \widetilde{\varphi}_{j, l}\right\rangle \varphi_{j, l}(x) \text { and } \mathscr{Q}_{j} f(x)=\sum_{l}\left\langle f, \widetilde{\psi}_{j, l}\right\rangle \psi_{j, l}(x) .
$$

Here $\widetilde{\varphi}_{j, l}$ and $\widetilde{\psi}_{j, l}$ are defined similarly as above. Such wavelets are called biorthogonal wavelets [7]. The dual functions also generate a multiresolution analysis with subspaces $\widetilde{V}_{j}$ and $\widetilde{W}_{j}$, which are different from the $V_{j}$ and $W_{j}$.

Taking the Fourier transform of Eqs. (1) and (2) yields

$$
\widehat{\varphi}(\omega)=H(\omega / 2) \widehat{\varphi}(\omega / 2) \text { with } H(\omega)=\sum_{k} h_{k} \mathrm{e}^{-\mathrm{i} k \omega}
$$

and

$$
\widehat{\psi}(\omega)=G(\omega / 2) \widehat{\psi}(\omega / 2), \quad \text { with } \quad G(\omega)=\sum_{k} g_{k} \mathrm{e}^{-\mathrm{i} k \omega} .
$$

Similar definitions and equations hold for the dual functions. A necessary condition for biorthogonality is then

$$
\forall \omega \in \mathbb{R}: \widetilde{M}(\omega) M^{\mathrm{T}}(\omega)=1
$$

where

$$
M(\omega)=\left[\begin{array}{ll}
H(\omega) & H(\omega+\pi) \\
G(\omega) & G(\omega+\pi)
\end{array}\right]
$$

and similarly for $\widetilde{M}(\omega)$. Now Cramer's rule says that

$$
\widetilde{G}(\omega)=-\frac{H(\omega+\pi)}{\Delta(\omega)} \text { and } \widetilde{H}(\omega)=\frac{G(\omega+\pi)}{\Delta(\omega)}
$$

where $\Delta(\omega)=\operatorname{det} M(\omega)$. The determinant $\Delta(\omega)$ does not vanish if and only if the wavelets generate complementary subspaces.

The definition of a multiresolution analysis implies that for $f(x) \in \mathrm{L}^{2}(\mathbb{R})$,

$$
\lim _{j \rightarrow \infty} \mathscr{P}_{j} f(x)=f(x) \text { and } f(x)=\sum_{j} \mathcal{Q}_{j} f(x)
$$

The error of the approximation in $V_{j}$ is denoted as $\mathscr{E}_{n} f(x)$, with

$$
\mathscr{E}_{n} f(x)=f(x)-\mathscr{P}_{n} f(x) \text { or } \mathscr{E}_{n} f(x)=\sum_{j=n}^{\infty} \mathscr{Q}_{j} f(x)
$$




\subsection{Orthogonal and semiorthogonal wavelets}

A scaling function and wavelet are called orthogonal if the set of functions $\left\{\varphi_{j, l} \mid\right.$ $\left.l \in \mathbb{Z}_{\}}\right\}$is an orthonormal basis of $V_{j}$ and if the set $\left\{\psi_{j, l} \mid j, l \in \mathbb{Z}\right\}$ is an orthonormal basis of $\mathrm{L}^{2}(\mathbb{R})$. This implies that $W_{j}$ is the orthogonal complement of $V_{j}$ in $V_{j+1}$. The projection operators onto $V_{j}$ and $W_{j}$ are orthogonal and give the optimal approximation in the $\mathrm{L}^{2}$-sense. The basis functions and dual functions coincide.

A biorthogonal basis is called semiorthogonal when the subspaces $W_{j}$ are the orthogonal complement of $V_{j}$ in $V_{j+1}$, but the basis of each subspace is not orthogonal. In this case the projection operators $\mathscr{P}_{j}$ and $\mathscr{Q}_{j}$ still are orthogonal projections. The dual multiresolution analysis subspaces $\widetilde{V}_{j}$ and $\widetilde{W}_{j}$ now coincide with $V_{j}$ and $W_{j}$. Essentially, the wavelets that live on different levels are orthogonal, while the ones that live on the same level are not. These wavelets are also called pre-wavelets. For a more detailed treatment and examples, see $[4,19,25,26]$.

\subsection{Wavelets and polynomials}

The moments of the scaling function and wavelet are defined as

$$
\mathscr{M}_{p}=\int_{-\infty}^{+\infty} x^{p} \varphi(x) d x \text { and } \mathscr{N}_{p}=\int_{-\infty}^{+\infty} x^{p} \psi(x) d x \quad \text { with } p \in \mathbb{N} .
$$

They are finite if $\varphi(x)$ and $\psi(x)$ belong to $\mathscr{D}^{p}$. For the moments of the dual functions we use the notations $\widetilde{\mathscr{C}}_{p}$ and $\widetilde{\mathbb{N}_{p}}$. The scaling functions cannot have a vanishing integral and are normalized with $\mathscr{\mathscr { C }}_{0}=\widetilde{\mathscr{C}_{0}}=1$. This implies that $H(0)=\widetilde{H}(0)=1$.

Let $N$ denote the number of vanishing moment of the dual wavelet,

$$
\widetilde{\mathscr{T}_{p}}=0 \text { for } 0 \leq p<N \text { and } \widetilde{\mathscr{N}_{N}} \neq 0
$$

This is the same as saying that $\widehat{\widetilde{\psi}}(\omega)$ has a root of multiplicity $N$ at $\omega=0$, and, since $\widehat{\widetilde{\varphi}}(0) \neq 0$, also that $\widetilde{G}(\omega)$ has a root of multiplicity $N$ at $\omega=0$. From Eq. (5), we see that this is equivalent to $H(\omega)$ having a root of multiplicity $N$ at $\omega=\pi$, which, by using Eq. (3), implies that

$$
\mathrm{i}^{p} \widehat{\varphi}^{(p)}(2 k \pi)=\delta_{k} \mathscr{C l}_{p} \text { for } 0 \leq p<N .
$$

Poisson's summation formula yields that

$$
\sum_{l}(x-l)^{p} \varphi(x-l)=\mathscr{M}_{p} \text { for } 0 \leq p<N
$$

By rearranging the last expression, we see that any polynomial with degree less than $N$ can be written as a linear combination of the functions $\varphi(x-l)$ with $l \in \mathbb{Z}$. Equation (6) is known as the Strang-Fix condition [15, 29, 31], and it implies that if $f \in \mathscr{C}^{N}$, then

$$
\left\|\mathscr{E}_{n} f(x)\right\|=O\left(h^{N}\right) \text { with } h=2^{-n} .
$$




\subsection{Contents of the paper}

In the first part of the paper we derive an asymptotical error expansion for $\mathscr{E}_{n} f(x)$ in terms of powers of $h\left(h=2^{-n}\right)$ assuming that $f(x)$ is a sufficiently differentiable function. Since the error decays as $O\left(h^{N}\right)$ we write the expansion as

$$
\mathscr{E}_{n} f(x)=\sum_{p=N}^{M} h^{p} T_{p-N}(x)+O\left(h^{M+1}\right) .
$$

We show that $T_{p-N}(x)$ consists of the $p$-th derivative of $f(x)$ multiplied with an oscillating function. We propose the name "monowavelets" for the oscillating functions. This generalizes an earlier result that was only valid for orthogonal and compactly supported wavelets [33]. In the case of spline wavelets we point out a connection between the monowavelets and Euler and Bernoulli splines.

In a second part we use the expansion to compare different multiresolution analyses that have the same number of dual vanishing moments and thus the same convergence rate. The factor $T_{0}(x)$ in the leading term is calculated and compared for different wavelet families. A first result is that it does not depend on how the complementary spaces $W_{j}$ are chosen. Consequently, for a fixed set of subspaces $V_{j}$, the leading term does not depend on the fact whether the wavelets are orthogonal or biorthogonal. We show that this is also true for the first $\widetilde{N}$ terms. In other words, whether a projection is orthogonal or not, does essentially not make a difference in case the function is smooth and the scale is sufficiently fine.

Finally, we show that $T_{0}(x)$ in the case of Daubechies' orthogonal wavelets is roughly $2^{N}$ times larger than in the case of spline wavelets (both orthogonal, semiorthogonal and biorthogonal because of the first result). Thus, in order to obtain an approximation with a certain accuracy, one needs, in general, one more level with Daubechies' orthogonal wavelets than with spline wavelets.

\section{Asymptotic error expansion}

In this section we derive $M+1$ terms of the asymptotic error expansion (where $M \geq N)$. Assume that $\psi(x), \widetilde{\psi}(x) \in \mathscr{D}^{M+1}, f(x) \in \mathscr{C}^{M+1}$, and that $f^{(l)}(x)$ is bounded for $l \leq M+1$. Recall that

$$
\mathcal{Q}_{n} f(x)=2^{n} \sum_{l}\left\langle f(y), \widetilde{\psi}\left(2^{n} y-l\right)\right\rangle \psi\left(2^{n} x-l\right)=\sum_{l} \mu_{n, l} \psi\left(2^{n} x-l\right) .
$$

Writing a Taylor formula around $y=x$ in the definition of $\mu_{n, l}$ yields (with $y$ as integration variable in the inner products):

$$
\begin{aligned}
\mu_{n, l}= & 2^{n}\left\langle f(y), \widetilde{\psi}\left(2^{n} y-l\right)\right\rangle \\
= & 2^{n}\left\langle\sum_{p=0}^{M} f^{(p)}(x) \frac{(y-x)^{p}}{p !}+f^{(M+1)}(\xi(x, y)) \frac{(y-x)^{M+1}}{(M+1) !}, \widetilde{\psi}\left(2^{n} y-l\right)\right\rangle \\
& \text { with } \xi(x, y) \text { between } x \text { and } y \\
= & \sum_{p=0}^{M} \frac{2^{n} f^{(p)}(x)}{p !}\left\langle(y-x)^{p}, \widetilde{\psi}\left(2^{n} y-l\right)\right\rangle+\rho_{n, l}(x),
\end{aligned}
$$


with

$$
\rho_{n, l}(x)=2^{n}\left\langle f^{(M+1)}(\xi(x, y)) \frac{(y-x)^{M+1}}{(M+1) !}, \widetilde{\psi}\left(2^{n} y-l\right)\right\rangle .
$$

As the derivatives of $f(x)$ are bounded and $\widetilde{\psi}(x)$ belongs to $\mathscr{D}^{M+1}$, all the inner products are finite. The dual wavelet has $N$ vanishing moments so that

$$
2^{n}\left\langle(y-x)^{p}, \widetilde{\psi}\left(2^{n} y-l\right)\right\rangle=0 \text { for } 0 \leq p<N,
$$

and thus the first $N$ terms of the summation over $p$ vanish. For $N \leq p \leq M$ we have, using the transformation $z=2^{n} y-l$, that

$$
\begin{aligned}
2^{n}\left\langle(y-x)^{p}, \widetilde{\psi}\left(2^{n} y-l\right)\right\rangle & =\left\langle(h z+h l-x)^{p}, \widetilde{\psi}(z)\right\rangle \\
& =h^{p} \sum_{s=0}^{p}\left(\begin{array}{l}
p \\
s
\end{array}\right) \widetilde{\widetilde{T}_{p-s}}\left(l-2^{n} x\right)^{s} .
\end{aligned}
$$

The last $N$ terms of this sum again vanish, so the upper bound of the summation over $s$ can be $p-N$. Thus,

$$
\gamma_{n, l}=\sum_{p=N}^{M} \frac{h^{p} f^{(p)}(x)}{p !} \sum_{s=0}^{p-N}\left(\begin{array}{l}
p \\
s
\end{array}\right) \widetilde{\mathscr{N}_{p-s}}\left(l-2^{n} x\right)^{s}+\rho_{n, l}(x) .
$$

Combining this expansion with Eq. (8) yields that

$$
\mathcal{Q}_{n} f(x)=\sum_{p=N}^{M} \frac{h^{p} f^{(p)}(x)}{p !} \sum_{s=0}^{p-N}\left(\begin{array}{l}
p \\
s
\end{array}\right) \widetilde{\widetilde{T}_{p-s}}(-1)^{s} \sigma_{s}\left(2^{n} x\right)+K_{n}(x) .
$$

Here $\sigma_{p}(x)$ is the first monowavelet, which is defined as

$$
\sigma_{p}(x)=\sum_{l}(x-l)^{p} \psi(x-l) \text { for } 0 \leq p<M-N,
$$

and $K_{n}(x)$ is given by

$$
K_{n}(x)=\sum_{l} \rho_{n, l}(x) \psi\left(2^{n} x-l\right)
$$

Next we show that $K_{n}(x)$ behaves like $O\left(h^{N}\right)$ :

$$
\begin{aligned}
&\left|K_{n}(x)\right| \leq \sum_{l}\left|\rho_{n, l}(x)\right|\left|\psi\left(2^{n} x-l\right)\right| \\
& \leq \frac{\left\|f^{(M+1)}(x)\right\|_{\infty}}{(M+1) !} 2^{n} \sum_{l}\left\langle|y-x|^{M+1},\left|\widetilde{\psi}\left(2^{n} y-l\right)\right|\right\rangle\left|\psi\left(2^{n} x-l\right)\right| \\
&=h^{M+1}\left\|f^{(M+1)}(x)\right\|_{\infty} \sum_{l}\left\langle|z+l-x / h|^{M+1},|\widetilde{\psi}(z)|\right\rangle|\psi(x / h-l)| \\
& \leq h^{M+1}\left\|f^{(M+1)}(x)\right\|_{\infty} . \\
&(M+1) !
\end{aligned}
$$




$$
\begin{aligned}
& \sum_{l}\left[\sum_{j=0}^{M+1} m_{j}|x / h-l|^{j}\left(\begin{array}{c}
M+1 \\
j
\end{array}\right)\right]|\psi(x / h-l)| \\
& \text { with } \left.m_{j}=\left\langle|z|^{M+1-j},|\widetilde{\psi}(z)|\right\rangle \text { (finite since } \widetilde{\psi}(x) \in \mathscr{D}^{M+1}\right) \\
\leq & h^{M+1}\left\|f^{(M+1)}(x)\right\|_{\infty} \cdot \max _{0 \leq j \leq M+1} m_{j} . \\
& \sum_{l}\left[\sum_{j=0}^{M+1}|x / h-l|^{j}\left(\begin{array}{c}
M+1 \\
j
\end{array}\right)\right]|\psi(x / h-l)| \\
= & h^{M+1}\left\|f^{(M+1)}(x)\right\|_{\infty} . \max _{0 \leq j \leq M+1} m_{j} . \\
& \left.\sum_{l}(\mid x / h-l) !+1\right)^{M+1}|\psi(x / h-l)| .
\end{aligned}
$$

Since this last summation over $l$ can be bounded independently of $x$ and $h$, it holds that $\left|K_{n}(x)\right| \leq C h^{M+1}$ with $C$ independent of $x$ and $n$. Now we combine the error expansions for $\mathscr{Q}_{n}$ into one for $\mathscr{E}_{n}$. Therefore, we define a new monowavelet $\sigma_{p}^{*}(x)$ and write

$$
\mathcal{Q}_{n} f(x)=\sum_{p=N}^{M} \frac{h^{p} f^{(p)}(x)}{p !} \sigma_{p-N}^{*}\left(2^{n} x\right)+K_{n}(x),
$$

with

$$
\sigma_{p}^{*}(x)=\sum_{s=0}^{p}\left(\begin{array}{c}
N+p \\
s
\end{array}\right) \widetilde{\widetilde{N}_{N+p-s}}(-1)^{s} \sigma_{s}(x) .
$$

So

$$
Q_{n+j} f(x)=\sum_{p=N}^{M} \frac{h^{p} f^{(p)}(x)}{p ! 2^{j p}} \sigma_{p-N}^{*}\left(2^{n+j} x\right)+K_{n+j}(x) .
$$

Finally, adding the projections $\mathscr{Q}_{n+j} f(x)$ yields the desired expansion,

$$
\mathscr{E}_{n} f(x)=\sum_{p=N}^{M} \frac{h^{p} f^{(p)}(x)}{p !} \tau_{p-N}\left(2^{n} x\right)+O\left(h^{M+1}\right) .
$$

Here $\tau_{p}(x)$ is the third monowavelet, which is defined as

$$
\tau_{p}(x)=\sum_{j=0}^{\infty} \frac{\sigma_{p}^{*}\left(2^{j} x\right)}{2^{j(p+N)}} .
$$

We conclude by saying that the general term of the expansions consists of: a power of $h$, the same order of derivative of $f(x)$, and a monowavelet. We can look at the monowavelet as the "oscillating" part and at the derivative as the "modulating" part. 


\section{Properties of monowavelets}

\subsection{Definition}

Recall that the monowavelet $\sigma_{p}(x)$ is defined as

$$
\sigma_{p}(x)=\sum_{l}(x-l)^{p} \psi(x-l) .
$$

It is the periodization of $x^{p} \psi(x)$ with period one. If $\psi(x) \in \mathscr{D}^{p}$, then the series in Eq. (12) converges uniformly on $[0,1]$ and $\sigma_{p}(x)$ is bounded. This can be seen using the Weierstrass $M$-test combined with the fact that $x^{p} \psi(x) \in \mathscr{D}^{0}$. One can check that this condition was always satisfied in the previous section. From the definition it follows that

$$
\int_{0}^{1} \sigma_{p}(x) d x=\mathscr{N}_{p} \text { for } p \in \mathbb{N}
$$

and thus the first $\widetilde{N}$ monowavelets have a vanishing mean.

The monowavelet $\tau_{p}(x)$ is defined as

$$
\tau_{p}(x)=\sum_{j=0}^{\infty} \frac{\sigma_{p}^{*}\left(2^{j} x\right)}{2^{j(p+N)}} .
$$

The series in Eq. (13) converges uniformly on [0,1], and the monowavelet is periodic with period one. Also,

$$
\sigma_{p}^{*}(x)=\tau_{p}(x)-\frac{\tau_{p}(2 x)}{2^{N+p}},
$$

and

$$
\int_{0}^{1} \tau_{p}(x) d x=\frac{2^{(N+p)}}{2^{(N+p)}-1} \int_{0}^{1} \sigma_{p}^{*}(x) d x=0 \quad \text { if } p<\tilde{N} .
$$

Again the first $\widetilde{N}$ monowavelets have a vanishing mean.

\subsection{Invariance}

There are obviously many possible choices for the wavelet $\psi(x)$ whose translates and dilates generate the same subspaces $W_{j}$. A trivial alternative would be $\psi(x-1)$. From its definition we see that the function $\sigma_{p}(x)$ depends on the particular choice for $\psi(x)$. This is not true for $\sigma_{p}^{*}(x)$ and $\tau_{p}(x)$. Writing Eq. (9) with $n=0$ in case $f(x)$ is the monomial $x^{p}$ with $p>N$ yields

$$
\mathcal{Q}_{0} x^{p}=\sum_{s=N}^{p}\left(\begin{array}{l}
p \\
s
\end{array}\right) x^{p-s} \sigma_{s-N}^{*}(x) .
$$

It follows that $\sigma_{p}^{*}(x)$ only depends on the multiresolution analysis subspaces $W_{j}$ and not on which particular functions $\psi_{j, k}(x)$ generate it. So $\sigma_{p}^{*}(x)$ is more characteristic for a multiresolution analysis than $\sigma_{p}(x)$. The same is true for $\tau_{p}(x)$ as

$$
\mathscr{E}_{0} x^{p}=(1-\mathscr{R}) x^{p}=\sum_{s=N}^{p}\left(\begin{array}{l}
p \\
s
\end{array}\right) x^{p-s} \tau_{s-N}(x) .
$$


These dependencies are studied in more detail in Sect. 8. Note that

$$
\tau_{0}(x)=x^{N}-\mathscr{B} x^{N} .
$$

This is the error of the approximation of the lowest degree monomial that cannot be approximated exactly. Equation (16) generalizes this to higher degree monomials.

This also explains the name "monowavelets". The monowavelets come from periodizing a monomial multiplied with a wavelet or from projecting down monomials. These techniques are also used in spline theory where the resulting functions are called monosplines [28].

\subsection{Fourier series}

Write the Fourier series of the monowavelet $\sigma_{p}(x)$ as

$$
\sigma_{p}(x)=\sum_{k} s_{p, k} e_{k}(x) \quad \text { with } e_{k}(x)=\exp (2 \pi \mathrm{i} k x),
$$

and

$$
s_{p, k}=\int_{0}^{1} \sigma_{p}(x) e_{k}(x) d x .
$$

Poisson's summation formula yields that

$$
s_{p, k}=\mathrm{i}^{p} \widehat{\psi}^{(p)}(2 k \pi) .
$$

The coefficients with even index can be written as

$$
s_{p, 2 k}=\mathrm{i}^{p} \widehat{\psi}^{(p)}(4 k \pi)=(\mathrm{i} / 2)^{p} \frac{d^{p}}{d \omega^{p}}[G(\omega) \widehat{\varphi}(\omega)]_{\omega=2 k \pi} .
$$

If $p<\widetilde{N}$, these terms vanish, since $2 k \pi$ is a root of order $\widetilde{N}$ of $G(\omega)$. So only the odd index terms, which are antisymmetric around $1 / 2$, remain so that

$$
\sigma_{p}(x+1 / 2)=-\sigma_{p}(x),
$$

or

$$
\sum_{l}(x+l / 2)^{p} \psi(x+l / 2)=0 \text { for } p<\tilde{N} .
$$

Equation (6) states that $2 k \pi, k \neq 0$ is a root of order $N$ of $\widehat{\varphi}(\omega)$. This, together with Eq. (17), yields that if $\widetilde{N} \leq p<N_{\text {tot }}=\widetilde{N}+N$, the $s_{p, 2 k}$ with $k \neq 0$ will vanish, or

$$
s_{p, 2 k}=\widehat{\mathrm{i}}^{p} \psi^{(p)}(0) \delta_{k}=\mathscr{N}_{p} \delta_{k} .
$$

So

$$
\sigma_{p}(x+1 / 2)+\sigma_{p}(x)=2 \cdot \mathscr{N}_{p},
$$

or

$$
\sum_{l}(x+l / 2)^{p} \psi(x+l / 2)=2 \cdot \mathscr{N}_{p} \text { for } \quad \widetilde{N} \leq p<N_{\text {tot }} .
$$

The monowavelet $\sigma_{p}^{*}(x)$ has Fourier series 


$$
\sigma_{p}^{*}(x)=\sum_{k} s_{p, k}^{*} e_{k}(x)
$$

with

$$
s_{p, k}^{*}=\sum_{j=0}^{p}\left(\begin{array}{c}
N+p \\
j
\end{array}\right) \widetilde{\mathscr{N}_{N}+p-j}(-1)^{j} s_{j, k} .
$$

Since they are defined as finite linear combinations of the $\sigma_{p}(x)$, the $\sigma_{p}^{*}(x)$ with $p<\widetilde{N}$ also have vanishing even coefficients in their Fourier series and thus satisfy,

$$
\sigma_{p}^{*}(x+1 / 2)=-\sigma_{p}^{*}(x) \text { for } p<\tilde{N}
$$

The monowavelet $\tau_{p}(x)$ has Fourier series

$$
\tau_{p}(x)=\sum_{k} t_{p, k} e_{k}(x)
$$

Writing the Fourier series of both sides of Eq. (14) gives

$$
t_{p, 2 k+1}=s_{p, 2 k+1}^{*} \quad \text { and } \quad t_{p, 2 k}=s_{p, 2 k}^{*}+\frac{t_{p, k}}{2^{N+p}},
$$

or, if $p<\tilde{N}$ and thus $s_{p, 2 k}^{*}=0$

$$
t_{p, k}=\frac{s_{p, 2 l+1}}{2^{m(N+p)}} \quad \text { with } \quad k=2^{m}(2 l+1)
$$

The transition from $\sigma_{p}(x)$ to $\tau_{p}(x)$ apparently corresponds to filling in the gaps at the even indices in the Fourier spectrum.

\subsection{Zeros}

Lemma 1. If $\psi(x)$ is continuous and in $\mathscr{D}^{p}$ with $p<\tilde{N}$, the monowavelets $\sigma_{p}(x)$ and $\sigma_{p}^{*}(x)$ have at least two zeros in the interval $[0,1)$.

Proof. If $\psi(x)$ is in $\mathscr{D}^{p}$, then the series in Eq. (12) converges uniformly and $\sigma_{p}(x)$ is continuous. Since $\sigma_{p}^{*}(x)$ is a finite linear combination, it is continuous too. The proof then immediately follows from Eqs. (18) and (20). Also, if $x_{0}$ is a root in [0,1), so is $\left(x_{0}+1 / 2\right) \bmod 1$.

Lemma 2. If $\psi(x)$ is continuous and in $\mathscr{D}_{p}$ with $p<\widetilde{N}$ and $N>1$, the monowavelet $\tau_{p}(x)$ has at least two zeros in the interval $[0,1)$.

Proof. The function $\tau_{p}(x)$ is defined as the limit of a uniformly convergent series of continuous functions, so it is continuous. We have, using Eqs. (20) and (21),

$$
\tau_{p}(0)=\tau_{p}(1)=\frac{2^{(N+p)}}{2^{(N+p)}-1} \sigma_{p}^{*}(0) \quad \text { and } \quad \tau_{p}(1 / 2)=-\frac{2^{(N+p)}-2}{2^{(N+p)}-1} \sigma_{p}^{*}(0) .
$$

This means we have at least two changes of sign. 


\subsection{Symmetry}

If the wavelet is even or odd,

$$
\psi(-x)=(-1)^{m} \psi(x),
$$

so are the monowavelets $\sigma_{p}(x)$ and $\sigma_{p}^{*}(x)$ and, more precisely,

$$
\sigma_{p}(-x)=(-1)^{m+p} \sigma_{p}(x) \text { and } \sigma_{p}^{*}(-x)=(-1)^{m+p} \sigma_{p}^{*}(x) \text {. }
$$

More generally, if the wavelet is (anti)symmetric around an integer $k$,

$$
\psi(2 k-x)=(-1)^{m} \psi(x),
$$

so is the monowavelet $\sigma_{p}^{*}(x)$,

$$
\sigma_{p}^{*}(-x)=(-1)^{m+p} \sigma_{p}^{*}(x)
$$

This is true because the function $\psi(x-k)$ generates the same space $W_{j}$ and thus gives rise to the same $\sigma_{p}^{*}(x)$ function while $\psi(x-k)$ is even or odd. Note that we cannot make a simple statement about $\sigma_{p}(x)$. The following statements regarding the zeros of $\sigma_{p}(x)$ (and consequently of $\left.\sigma_{p}^{*}(x)\right)$ and $\tau_{p}(x)$, for $p<\tilde{N}$ hold:

- If $\sigma_{p}(x)$ is odd, it has zeros at the integers because of $\sigma_{p}(0)=0$ and the periodicity. It then also has zeros at the integers $+1 / 2$ because of Eq. (18).

- If $\sigma_{p}(x)$ is even, this combined with Eq. (18) yields $\sigma_{p}(x)=-\sigma_{p}(1 / 2-x)$. It thus has zeros at the integers $+1 / 4$, and again because of Eq. (18) also at the integers $+3 / 4$.

- If $\tau_{p}(x)$ is odd, it has zeros at the half integers; if it is even we cannot tell more about the position of its zeros this easily.

\subsection{Connection with scaling function}

The relationship between $\sigma_{p}(x)$ for $p<N_{\text {tot }}$ and the scaling function can be written more explicitly using the Zak transform. Remember that the Zak transform of a function $f(x) \in \mathrm{L}^{2}(\mathbb{R})$ is defined as $[16,17]$ :

$$
(\mathscr{Z} f)(x, \omega)=\sum_{l} \mathrm{e}^{-\mathrm{i} \omega l} f(x+l) \text { for } \quad x, \omega \in \mathbb{R}
$$

and satisfies

$$
(\mathscr{Z} f)(x, \omega)=\sum_{k} \widehat{f}(\omega+2 \pi k) \mathrm{e}^{\mathrm{i}(\omega+2 \pi k) x} .
$$

Define now:

$$
\gamma_{p}=\mathrm{i}^{p} G^{(p)}(\pi)=\sum_{k} k^{p}(-1)^{k} g_{k} .
$$

Then 


$$
\begin{aligned}
s_{p, 2 k+1} & =\mathrm{i}^{p} \frac{d^{p}}{d \omega^{p}}[G(\omega / 2) \widehat{\varphi}(\omega / 2)]_{\omega=(2 k+1) 2 \pi} \\
& =\left(\begin{array}{l}
\mathrm{i} \\
2
\end{array}\right)^{p} \sum_{s=0}^{p}\left(\begin{array}{l}
p \\
s
\end{array}\right) G^{(p-s)}(\pi) \widehat{\varphi}^{(s)}((2 k+1) \pi) \\
& =2^{-p} \sum_{s=0}^{p}\left(\begin{array}{l}
p \\
s
\end{array}\right) \gamma_{p-s} \mathrm{i}^{s} \widehat{\varphi}^{(s)}((2 k+1) \pi)
\end{aligned}
$$

Now, for $p<\widetilde{N}$,

$$
\begin{aligned}
\sigma_{p}(x) & =\left(\mathscr{Z} x^{p} \psi\right)(x, 0) \\
& =\sum_{k} s_{p, 2 k+1} e_{2 k+1}(x) \\
& =2^{-p} \sum_{s=0}^{p}\left(\begin{array}{l}
p \\
s
\end{array}\right) \gamma_{p-s} \sum_{k} \mathrm{i}^{s} \widehat{\varphi}^{(s)}((2 k+1) \pi) e_{2 k+1}(x) \\
& =2^{-p} \sum_{s=0}^{p}\left(\begin{array}{l}
p \\
s
\end{array}\right) \gamma_{p-s}\left(\mathscr{Z} x^{s} \varphi\right)(2 x, \pi) \\
& =2^{-p} \sum_{s=0}^{p}\left(\begin{array}{l}
p \\
s
\end{array}\right) \gamma_{p-s} \sum_{l}(-1)^{l}(2 x-l)^{s} \varphi(2 x-l) .
\end{aligned}
$$

For $\tilde{N} \leq p<N_{\text {tot }}$ holds that

$$
\sigma_{p}(x)=\mathscr{N}_{p}+2^{-p} \sum_{s=0}^{p}\left(\begin{array}{l}
p \\
s
\end{array}\right) \gamma_{p-s} \sum_{l}(-1)^{l}(2 x-l)^{s} \varphi(2 x-l) .
$$

In case $p=0$ we have that

$$
s_{0,2 k+1}=G(\pi) \widehat{\varphi}((2 k+1) \pi)=\widehat{\varphi}((2 k+1) \pi),
$$

and

$$
\sigma_{0}(x)=\sum_{l}(-1)^{l} \varphi(2 x-l)=2 \sum_{l} \varphi(2 x-2 l)-1 .
$$

\section{Spline monowavelets}

In this section we study the monowavelets in case the scaling function and wavelet are spline functions. Therefore, we first introduce Euler and Bernoulli polynomials and splines.

\subsection{Euler and Bernoulli polynomials and splines}

A sequence of polynomials $V_{m}(x), m \in \mathbb{N}$, is an Appell sequence if $V_{m}(x)$ is a polynomial of strict degree $m$ and

$$
V_{m}^{\prime}(x)=m V_{m-1}(x) .
$$



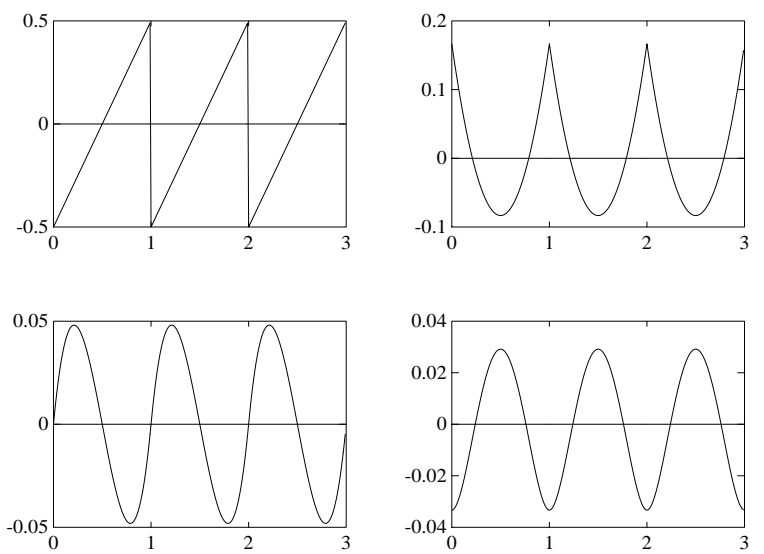

Fig. 1. $\mathbf{B}_{m}(x)$ for $m=1,2,3,4$

The Euler and Bernoulli polynomials are two Appell sequences, denoted with $E_{m}(x)$ and $B_{m}(x)$ respectively, that satisfy $[1,14]$

$$
\frac{2 \mathrm{e}^{x z}}{\mathrm{e}^{z}+1}=\sum_{n} \frac{E_{m}(x)}{m !} z^{m} \text { for }|z|<2 \pi,
$$

and

$$
\frac{z \mathrm{e}^{x z}}{\mathrm{e}^{z}-1}=\sum_{n} \frac{B_{m}(x)}{m !} z^{m} \text { for }|z|<\pi .
$$

The first elements of the sequences are

$$
\begin{aligned}
& E_{0}(x)=1 \\
& E_{1}(x)=x-1 / 2 \\
& E_{2}(x)=x^{2}-x \\
& E_{3}(x)=x^{3}-3 / 2 x^{2}+1 / 4 \\
& E_{4}(x)=x^{4}-2 x^{3}+x,
\end{aligned}
$$$$
B_{0}(x)=1
$$$$
B_{1}(x)=x-1 / 2
$$$$
\text { and } \quad B_{2}(x)=x^{2}-x+1 / 6
$$$$
B_{3}(x)=x^{3}-3 / 2 x^{2}+1 / 2 x \text {. }
$$$$
B_{4}(x)=x^{4}-2 x^{3}+x^{2}-1 / 30 \text {. }
$$

The Bernoulli polynomials satisfy

$$
B_{m}(x+1)-B_{m}(x)=m x^{m-1},
$$

and consequently

$$
B_{m}^{(p)}(0)=B_{m}^{(p)}(1) \text { for } 0 \leq p \leq m-2 .
$$

This means that, if we define a one-periodic function $\mathbf{B}_{m}(x)$ as

$$
\mathbf{B}_{m}(x)=B_{m}(x-[x]),
$$

then this is a periodic spline of order $m+1$ with integer knots that belongs to $\mathscr{C}^{m-2}$. It is called the Bernoulli periodic spline [21]. The first four are shown in Fig. 1. Its Fourier series for $m \geq 1$ is

$$
\mathbf{B}_{m}(x)=-\frac{m !}{(2 \pi \mathbf{i})^{m}} \sum_{k}^{\prime} \frac{e_{k}(x)}{k^{m}} .
$$



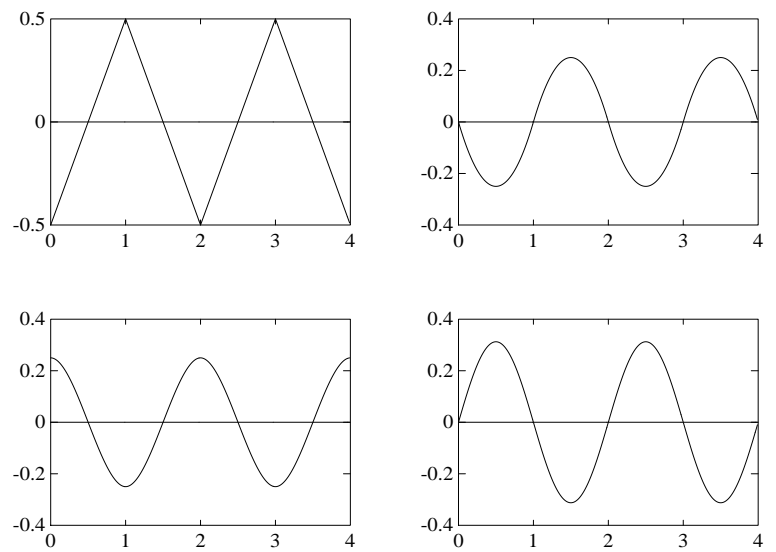

Fig. 2. $\mathbf{E}_{m}(x)$ for $m=1,2,3,4$

The prime indicates that the term with $k=0$ is omitted. The function $\mathbf{B}_{m}(x)$ has the same parity (even/odd) as $m$.

Something similar is possible for the Euler polynomials. They satisfy

$$
E_{m}(x+1)+E_{m}(x)=2 x^{m},
$$

and consequently

$$
E_{m}^{(p)}(0)=-E_{m}^{(p)}(1) \text { for } 0 \leq p \leq m-1 .
$$

This means that if we define a two-periodic function $\mathbf{E}_{m}(x)$ as

$$
\mathbf{E}_{m}(x)=(-1)^{[x]} E_{m}(x-[x]),
$$

then this is a periodic spline of order $m+1$ with integer knots that belongs to $\mathscr{C}^{m-1}$. It is called the Euler periodic spline [21, 28]. The first four are shown in Fig. 2. The function $\mathbf{E}_{m}(x)$ has the opposite parity (even/odd) as $m$. Also,

$$
\mathbf{E}_{m}(j+m / 2)=(-1)^{j} \epsilon_{m} .
$$

It is sometimes normalized so that $\epsilon_{m}=1$, see $[3,28]$. It is then the cardinal spline interpolant of the sequence $y_{k}=(-1)^{k}$. Its Fourier series for $m>1$ is

$$
\mathbf{E}_{m}(x)=\frac{2 m !}{(\pi \mathrm{i})^{m+1}} \sum_{k} \frac{e_{2 k+1}(x / 2)}{(2 k+1)^{m+1}} .
$$

\subsection{Spline wavelets}

We consider the multiresolution analysis where $V_{0}$ is the space of piecewise polynomials of degree $m-1$ with integer knots that belong to $\mathscr{C}^{m-2}$. Note that this implies that $N=m$. From the dual multiresolution analysis we only require that $\widetilde{N}>0$. The dual scaling function and wavelet need not be splines. We supply the notation with an extra superscript $(m)$. A possible choice of scaling function is $\varphi^{(m)}(x)=N_{m}(x)$, the cardinal B-spline of order $m$. We know that 


$$
\widehat{N}_{m}(\omega)=\left(\frac{1-\mathrm{e}^{-\mathrm{i} \omega}}{\mathrm{i} \omega}\right)^{m}
$$

so that (from Eqs. (22) and (23))

$$
s_{0,2 k+1}^{(m)}=\left(\begin{array}{c}
2 \\
\mathrm{i} \pi
\end{array}\right)^{m} \frac{1}{(2 k+1)^{m}} \quad \text { and } t_{0, k}^{(m)}=\widetilde{\mathscr{T}_{m}^{(m)}}\left(\begin{array}{c}
2 \\
\mathrm{i} \pi
\end{array}\right)^{m} \frac{1}{k^{m}} .
$$

We will show in Sect. 8 that, for all possible dual wavelets,

$$
\widetilde{\mathscr{N}_{m}^{(m)}}=-\frac{m !}{2^{2 m}}
$$

This implies that

$$
\sigma_{0}^{(m)}(x)=\frac{2^{m-1}}{(m-1) !} \mathbf{E}_{m-1}(2 x) \text { and } \tau_{0}^{(m)}(x)=\mathbf{B}_{m}(x)
$$

The $\sigma_{p}(x)$ and $\tau_{p}(x)$ functions with $p>0$ here are also one-periodic splines of order $m$. Analytic expressions can be obtained, but the derivation is quite technical and is omitted here. We refer to [32] for details. We only include the main result, which states that

$$
\tau_{p}^{(m)}(x)=(-1)^{p}\left(\begin{array}{c}
m \\
p
\end{array}\right) \mathbf{B}_{m+p}(x) \text { for } p<N_{\text {tot }} .
$$

The fact that we have an analytic expression for the monowavelets here is extremely useful in convergence acceleration algorithms as described in [33].

Remark 1. Equation (13) for $p=0$ yields the following relationship between Euler and Bernoulli splines:

$$
\mathbf{B}_{m}(x)=-\frac{m}{2^{m+1}} \sum_{i=0}^{\infty} \frac{\mathbf{E}_{m-1}\left(2^{i+1} x\right)}{2^{m i}}
$$

Something similar is also true for the polynomials, as

$$
B_{m}(x)=-\frac{m}{2^{m+1}} \sum_{i=0}^{k} \frac{E_{m-1}\left(2^{i+1} x\right)}{2^{m i}}+\frac{B_{m}\left(2^{k+1} x\right)}{2^{m(k+1)}}
$$

and this is an iterated version of [1, Eq. (23.1.27)].

Remark 2. In [30, pp. 147-151], Strang and Fix construct an asymptotical error analysis for the projection in the space spanned by piecewise linear finite elements. This result coincides with the one presented here in case $m=2$ and $M=2$. 


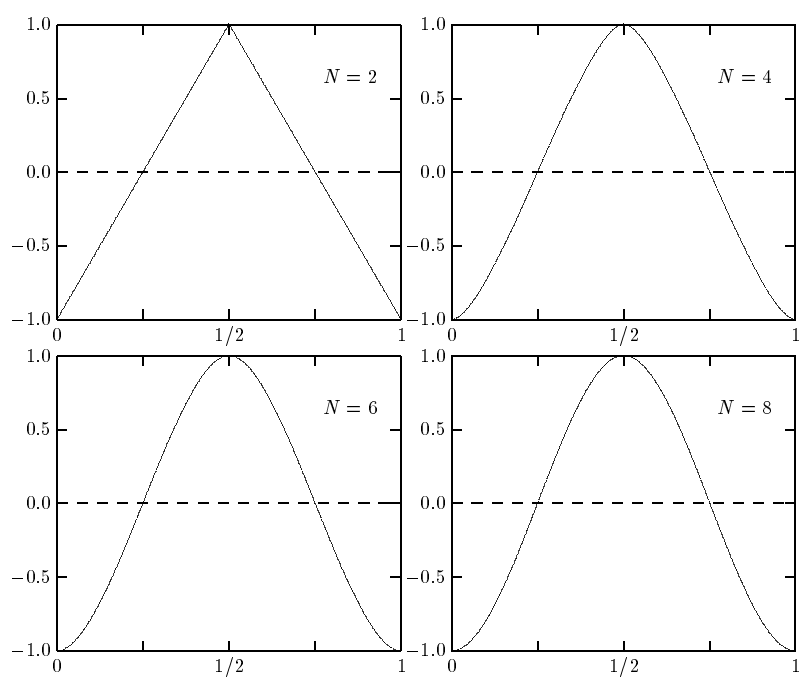

Fig. 3. $\sigma_{0}(x)$ in the case of Deslauriers-Dubuc with $N=2,4,6,8$

\section{Other examples of monowavelets}

- For the Daubechies orthogonal wavelets with compact support $[8,9,10]$ (the extremal phase, closest-to-linear phase and coiflets), these functions were studied in [33]. Some of the graphs can be found there too.

- The Deslauriers-Dubuc scaling functions and wavelets can be constructed as autocorrelation functions of the Daubechies orthogonal scaling functions and wavelets $[11,12,13,27]$. The $\sigma_{0}(x)$ for the cases $N=2,4,6,8$ are shown in Fig. 3 .

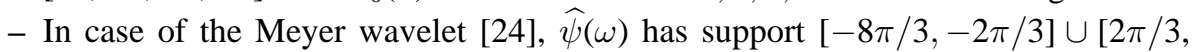
$8 \pi / 3$ ], so $\widehat{\psi}(2 k \pi)$ is identically zero for $|k| \neq 1$. We have that $\widehat{\psi}( \pm 2 \pi)=-\sqrt{ } 2 / 2$, so that

$$
\sigma_{0, \text { Meyer }}(x)=-\sqrt{2} \cos (2 \pi x) .
$$

The Meyer wavelet has faster than polynomial decay such that all the functions $\sigma_{p}(x)$ are defined. They will be all of the form

$$
\sigma_{p, \text { Meyer }}(x)=a_{p} \sin \left(2 \pi x-b_{p}\right) .
$$

The asymptotical error expansion, however, does not make sense in this case since all the moments of this wavelet vanish $(N=\infty)$.

- For the Shannon wavelet, where

$$
\psi_{\text {Shannon }}(x)=\frac{\sin (2 \pi x)-\sin (\pi x)}{\pi x},
$$

one can see that

$$
\sum_{l} \psi_{\text {Shannon }}(x-l)
$$

diverges. Note that the Shannon wavelet is not in $\mathscr{D}^{0}$. 
For the Meyer wavelet, which has infinitely many vanishing moments, the $\sigma_{p}(x)$ monowavelet is a sine. In the case of spline wavelets, it is easily seen from their Fourier series that the Euler and Bernoulli periodic splines converge to a sine or cosine as $m$ goes to infinity. For the different Daubechies families, the monowavelets seem to converge to a sine function as $N$ goes to infinity. So it appears that as the number of vanishing moments goes to infinity, the monowavelets converge to sine functions.

\section{Interpolation}

The leading term of the expansion looks like

$$
h^{N} \tau_{0}(x / h) f^{(N)}(x) N ! .
$$

Remember that we stated that it consists of an oscillating and a modulating part. The modulating part is given by the envelopes

$$
h^{N} f^{(N)}(x) \tau_{\max } \quad \text { and } \quad h^{N} f^{(N)}(x) \tau_{\min },
$$

where

$$
\tau_{\max }=\max _{x \in[0,1]} \tau_{0}(x) N ! \text { and } \tau_{\min }=\min _{x \in[0,1]} \tau_{0}(x) N ! .
$$

The first term oscillates between these two envelopes. As a result of Lemma 2 this function has at least $2^{n+1}$ zeros per unit length. This leads to the following theorem:

Theorem 1. If $f(x)$ is sufficiently smooth, the approximation $\mathscr{P}_{n} f(x)$ asymptotically interpolates $f(x)$ in at least $2^{n+1}$ points per unit length.

The "asymptotically" here means that one can always find a large enough $n$ such that the interpolating properties hold. Essentially, one needs to take $n$ so that the remaining terms do not influence the zeros of the first term. The examples of Sect. 9 will show that $n$ need not be very large. Note that the number of interpolation points is twice the number of basis functions. The interpolation points $z_{k}$, where $\mathscr{P}_{n} f\left(z_{k}\right)=f\left(z_{k}\right)$, satisfy

$$
z_{2 k}=\left(x_{1}+k\right) h+O\left(h^{2}\right) \text { and } z_{2 k+1}=\left(x_{2}+k\right) h+O\left(h^{2}\right),
$$

where $x_{1}$ and $x_{2}$ are zeros of $\tau_{0}(x)$ in $[0,1)$.

\section{Comparison of multiresolution analyses}

The error expansion can be used to compare different multiresolution analyses. The error decays as $O\left(h^{N}\right)$ and the constant in front of this factor is given by

$$
T_{0}(x)=\tau_{0}(x / h) \frac{f^{(N)}(x)}{N !} .
$$

For sufficiently small $h$ the leading term of the expansion provides a sufficiently accurate approximation of the error. To compare different multiresolution analyses we therefore look at

$$
A_{N}=\left\|\tau_{0}(x)\right\|_{\infty},
$$

because 


$$
\left\|\mathscr{E}_{n} f(x)\right\|_{p} \approx h^{N}\left\|T_{0}(x)\right\|_{p} \leq \frac{A_{N} h^{N}}{N !}|f(x)|_{N, p} .
$$

Here the Sobolev seminorm is defined as

$$
|f(x)|_{N, p}=\left\|f^{(N)}(x)\right\|_{p} .
$$

\subsection{Multiresolution analyses with different $V_{j}$ subspaces}

A first possibility is to compare different multiresolution analyses that have the same number of vanishing dual wavelet moments. The order of convergence of the wavelet approximation evidently is the same. Therefore, we compare the numerical value of the constant $A_{N}$. Table 1 gives $A_{N}$ as a function of $N$ for the Daubechies orthogonal wavelets, spline wavelets, and Deslauriers-Dubuc wavelets. As we will see in the next section, $A_{m}$ is the same for all spline wavelets (orthogonal, biorthogonal or semiorthogonal) of order $m$. The spline wavelets have by far the smallest constants.

The ratio between $A_{N}$ for Daubechies' orthogonal wavelets and spline wavelets behaves roughly like $2^{N}$. Consequently, an approximation using splines at a certain level $n$ yields roughly the same error as an approximation using Daubechies' orthogonal scaling functions at level $n+1$. In other words, in order to obtain an approximation with a certain numerical error, one needs, in general, one more level with Daubechies' orthogonal wavelets than with spline wavelets. Remember that one extra level doubles the amount of work.

Note: The fact that the first non-vanishing dual wavelet moment plays a role in comparing errors in the discrete case was also pointed out in [20].

Table 1. $A_{N}$ for different wavelet families

$\begin{array}{rrrrrr}N & \begin{array}{r}\text { extremal } \\ \text { phase }\end{array} & \begin{array}{r}\text { closest-to- } \\ \text { linear phase }\end{array} & \text { coiflet } & \text { spline } & \begin{array}{r}\text { Deslauriers- } \\ \text { Dubuc }\end{array} \\ 1 & 0.500 & 0.500 & & 0.5000 & \\ 2 & 0.500 & 0.500 & 0.641 & 0.1667 & 0.1667 \\ 3 & 0.597 & 0.597 & & 0.0481 & \\ 4 & 0.865 & 0.915 & 0.856 & 0.0333 & 0.3000 \\ 5 & 1.904 & 1.918 & & 0.0244 & \\ 6 & 5.109 & 5.701 & 4.899 & 0.0238 & 1.7857 \\ 7 & 18.169 & 18.044 & & 0.0261 & \\ 8 & 70.927 & 71.865 & 59.436 & 0.0333 & 21.6176 \\ 9 & 310.398 & 303.921 & & 0.0476 & \\ & & & & & \end{array}$

\subsection{Multiresolution analyses with the same $V_{j}$ subspaces}

Another idea is to compare $A_{N}$ for a multiresolution analysis with fixed $V_{j}$ subspaces, but different $W_{j}$ subspaces and consequently different projection operators. Typically, we want to compare biorthogonal bases with (semi)orthogonal ones. Recall that in the latter case the projection operators are orthogonal and yield best approximations in the $\mathrm{L}^{2}$ sense.

In order to compare the error expansion for different families of wavelets, we first need to study some dependencies in a multiresolution analysis more carefully. Here we always use the normalizations 


$$
\widehat{\varphi}(0)=1 \text { and } G(\pi)=1,
$$

to avoid non-uniqueness. The following dependencies now hold in any multiresolution analysis:

- Given a scaling function, the subspaces $V_{j}$ are uniquely determined by

$$
V_{j}=\operatorname{clos} \operatorname{span}\left\{\varphi_{j, l}(x) \mid l \in \mathbb{Z}\right\} .
$$

On the other hand, given the subspaces $V_{j}$, infinitely many scaling functions exist that generate these spaces. More precisely, if $\varphi(x)$ is a such a function, any function $\varphi^{*}(x)$ with

$$
\widehat{\varphi}^{*}(\omega)=A(\omega) \widehat{\varphi}(\omega),
$$

where $A(\omega)$ is a bounded $2 \pi$-periodic function that does not vanish and $A(0)=1$, generates the same subspaces $V_{j}$. Moreover, any function generating the same subspaces is of this form.

- A similar statement holds for the wavelet $\psi(x)$ and the subspaces $W_{j}$.

- Given the subspaces $W_{j}$, the subspaces $V_{j}$ are uniquely determined by

$$
V_{j}=\bigoplus_{i=-\infty}^{j-1} W_{i} .
$$

On the other hand, if the $V_{j}$ are given, infinitely many choices for complementary spaces $W_{j}$ are possible, one choice being the orthogonal complements.

- Given the spaces $W_{j}$, the $\widetilde{W}_{j}$ are uniquely determined by the fact that

$$
W_{j} \perp \widetilde{W}_{j^{\prime}} \quad \text { if } j \neq j^{\prime} \text { and } \bigoplus_{j} \widetilde{W}_{j}=\mathrm{L}^{2}(\mathbb{R}) .
$$

Figure 4 shows these dependencies in a graph.

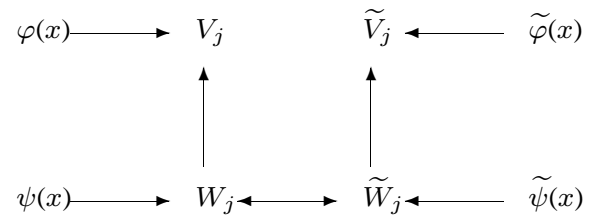

Fig. 4. Dependencies in a multiresolution analysis

For each characteristic of a multiresolution analysis we now can define its dependency: $V$-dependent, $\varphi$-dependent, $W$-dependent, or $\psi$-dependent. Something is called $\varphi$-dependent if it depends on the specific choice of scaling function. The other dependencies are defined similarly. We always use the most characteristic dependency. By looking at the dependency graph we see that something that is $V$-dependent is also $\varphi$-dependent, but we only use the term $\varphi$-dependent for something that is not $V$-dependent. In other words, something that is $V$-dependent does not change if the scaling function $\varphi(x)$ is replaced with a function $\varphi^{*}(x)$ that generates the same $V_{j}$ spaces. In order to become more familiar with this terminology, we illustrate it with some examples. 
- Anything that is $V$-dependent is also $W$-dependent.

- The projection $Q_{n} f(x)$ is $W$-dependent.

- The projection $\mathscr{P}_{n} f(x)$ is $W$-dependent too, as

$$
\mathscr{P}_{n}=\sum_{j=-\infty}^{n-1} Q_{j}
$$

- The number of vanishing dual wavelet moments $(N)$ is $V$-dependent.

- The $\sigma_{0}(x)$ monowavelet is $\varphi$-dependent because of Eq. (24).

- The $\sigma_{p}^{*}(x)$ and $\tau_{p}(x)$ monowavelet are, in general, $W$-dependent because of Eqs. (15) and (16).

- Anything that is $\varphi$-dependent and $W$-dependent at the same time is $V$-dependent.

We extend these properties with the following lemmas:

Lemma 3. The first non-vanishing dual wavelet moment is $V$-dependent and, more precisely,

$$
\widetilde{\mathscr{N}_{N}}=-(\mathrm{i} / 2)^{N} H^{(N)}(\pi)=(-1 / 2)^{N} \sum_{k}(-)^{k} k^{N} h_{k} \text {. }
$$

Proof. We first prove that it is $\varphi$-dependent. Pick, therefore, a scaling function $\varphi(x)$ that generates the $V_{j}$. Recall that

$$
\widetilde{G}(\omega)=-\frac{H(\omega+\pi)}{\Delta(\omega)}
$$

with

$$
\Delta(\omega)=H(\omega) G(\omega+\pi)-H(\omega+\pi) G(\omega) .
$$

Now,

$$
\widetilde{\mathscr{T}_{N}}=\mathrm{i}^{N} \widehat{\widetilde{\psi}}^{(N)}(0)=\mathrm{i}^{N} \frac{d^{N}}{d \omega^{N}}[\widetilde{G}(\omega / 2) \widehat{\widetilde{\varphi}}(\omega / 2)]_{\omega=0},
$$

and, since $\omega=0$ is a root of multiplicity $N$ of $\widetilde{G}(\omega)$,

$$
\widetilde{\mathscr{N}_{N}}=(\mathrm{i} / 2)^{N} \widetilde{G}^{(N)}(0) \text {. }
$$

Since $\omega=\pi$ is a root of multiplicity $N$ of $H(\omega)$, it holds that

$$
\widetilde{G}^{(N)}(0)=-\frac{H^{(N)}(\pi)}{\Delta(0)} .
$$

The fact that $\Delta(0)=1$ now yields the $\varphi$-dependency.

To prove that $\widetilde{\mathscr{T}_{N}}$ is $V$-dependent, take a different scaling function,

$$
\widehat{\varphi}^{*}(\omega)=A(\omega) \widehat{\varphi}(\omega),
$$

which has

$$
H^{*}(\omega)=\frac{A(2 \omega)}{A(\omega)} H(\omega) .
$$

As $A(0)=1$, and $\pi$ is a zero of order $N$ of $H(\omega)$, it holds that

$$
H^{*(N)}(\pi)=H^{(N)}(\pi) / A(\pi),
$$

which yields the same first non-vanishing dual wavelet moment. 
Lemma 4. The leading term of the error expansion is $V$-dependent.

Proof. The monowavelet $\sigma_{0}(x)$ and $\widetilde{\mathscr{N}_{N}}$ are $\varphi$-dependent, so $\sigma_{0}^{*}(x)=\widetilde{\mathscr{N}_{N}} \sigma_{0}(x)$ is $\varphi$-dependent too. As $\sigma_{0}^{*}(x)$ was already $W$-dependent, it is thus $V$-dependent. So also $\tau_{0}(x)$ and consequently the leading term of the expansion are $V$-dependent.

Note: If the subspace $V_{0}$ is the space of the piecewise polynomials of degree $m-1$ with integer knots that belong to $\mathscr{C}^{m-2}$, we can take $\varphi^{(m)}(x)$ to be the B-spline $N_{m}(x)$, so

$$
H(\omega)=\left(\begin{array}{c}
1+\mathrm{e}^{-\mathrm{i} \omega} \\
2
\end{array}\right)^{m}
$$

such that

$$
\widetilde{\mathscr{T}_{m}^{(m)}}=-\frac{m !}{2^{2 m}}
$$

and

$$
A_{m}=\left\|\mathbf{B}_{m}(x)\right\|_{\infty} .
$$

In the case $m$ is even, we have a simple expression since

$$
A_{2 n}=\left\|\mathbf{B}_{2 n}(x)\right\|_{\infty}=\left|B_{2 n}\right|,
$$

where $B_{2 n}$ is the $2 n$th Bernoulli number. The leading term of the expansion is exactly the same for Battle-Lemarié orthogonal spline wavelets [2, 22], Cohen-DaubechiesFeauveau biorthogonal spline wavelets [7], and Chui-Wang semiorthogonal spline wavelets $[4,5,6]$.

The dependency of the higher order terms is studied in the following lemmas.

Lemma 5. Given $\widetilde{N}$, the first $N_{\text {tot }}=N+\widetilde{N}$ moments of the dual scaling function are $\varphi$-dependent.

Proof. Since $\widetilde{\varphi}(x)$ is a dual function, it holds that

$$
\sum_{k} \widehat{\widetilde{\varphi}}(\omega+k 2 \pi) \widehat{\varphi}(\omega+k 2 \pi)=1 .
$$

Taking the $p$ th derivative of this expression at $\omega=0$ yields

$$
\sum_{k} \sum_{s=0}^{p}\left(\begin{array}{l}
p \\
s
\end{array}\right) \widehat{\widetilde{\varphi}}^{(s)}(k 2 \pi) \widehat{\varphi}^{(p-s)}(k 2 \pi)=0 .
$$

Now, since

$$
\mathrm{i}^{p} \widehat{\varphi}^{(p)}(2 k \pi)=\mathscr{M}_{p} \delta_{k} \text { for } 0 \leq p<N,
$$

and

$$
\mathrm{i}^{p} \widehat{\widetilde{\varphi}}^{(p)}(2 k \pi)=\widetilde{\mathscr{U}_{p}} \delta_{k} \text { for } 0 \leq p<\widetilde{N}
$$

it holds that

$$
\sum_{k} \widehat{\widetilde{\varphi}}^{(l)}(k 2 \pi) \widehat{\varphi}^{(m)}(k 2 \pi)=\widehat{\widehat{\varphi}}^{(l)}(0) \widehat{\varphi}^{(m)}(0)
$$

for $0 \leq l<\widetilde{N}$ or $0 \leq m<N$. The terms for $k \neq 0$ in Eq. (27) thus vanish if $s<\widetilde{N}$ or $p-s<N$. Consequently, if $p<N_{\text {tot }}$, then 


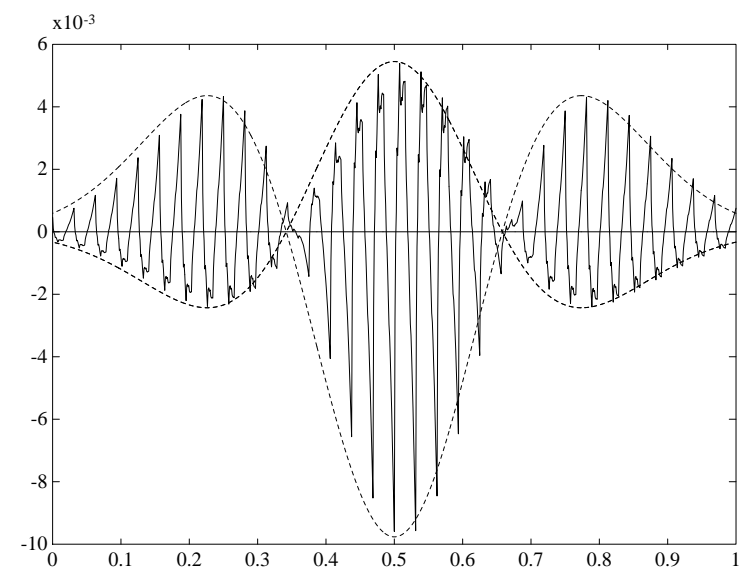

Fig. 5.

$$
\sum_{s=0}^{p}\left(\begin{array}{l}
p \\
s
\end{array}\right)(-1)^{s} \cdot \widetilde{\mathscr{C}_{s}} \mathscr{M}_{p-s}=0 \text { for } 0<p<N_{\text {tot }}
$$

These relations show that the first $N_{\text {tot }}$ moments of the dual scaling function only depend on the first $N_{\text {tot }}$ moments of the scaling function.

Note: a similar statement holds for the first $N_{\text {tot }}$ discrete moments of the sequence $\left\{h_{k}\right\}$.

Lemma 6. The functions $\mathcal{Q}_{n} x^{p}$ and $\mathscr{E}_{n} x^{p}$ are $V$-dependent if $p<N_{\text {tot. }}$

Proof. It follows from Lemma 5 that the function $\mathscr{P}_{n} x^{p}$ is $V$-dependent. The proof then immediately follows from the fact that $\mathscr{E}_{n}=1-\mathscr{P}_{n}$ and $\mathscr{Q}_{n}=\mathscr{P}_{n+1}-\mathscr{P}_{n}$.

Lemma 7. The functions $\sigma_{p}^{*}(x)$ and $\tau_{p}(x)$ are $V$-dependent if $p<\tilde{N}$.

Proof. From Lemma 6, Eq. (15), and Eq. (16).

These lemmas can be combined into the following theorem:

Theorem 2. The first $\tilde{N}$ terms of the error expansion are $V$-dependent.

So we can conclude that for the approximation of a smooth function on a small scale it does not really matter how the spaces $W_{j}$ are chosen. The outcome that orthogonal and biorthogonal projections almost give the same result might look surprising at first sight, but one has to keep in mind that it only holds for smooth functions.

\section{Numerical examples}

We implemented a computer program that calculates the operator $\mathscr{P}_{n} f(x)$ from samples of a function $f(x)$. It employs quadrature formula to approximate the inner products and a subdivision scheme to evaluate the basis functions. The error of these 


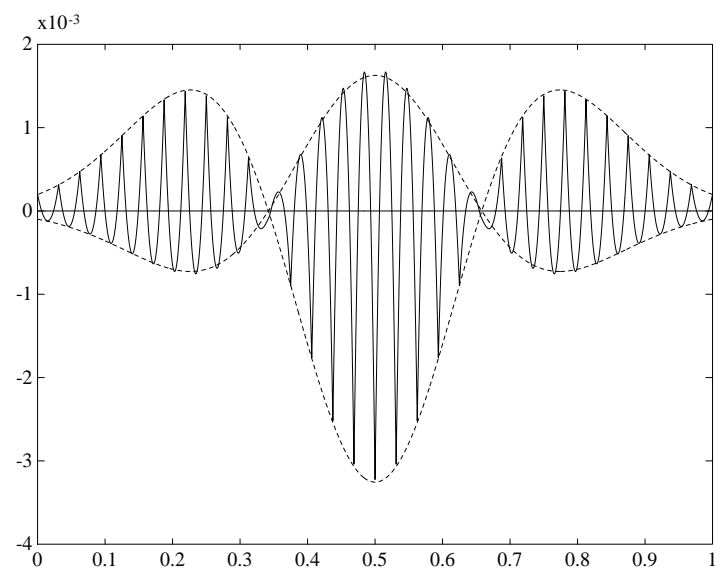

Fig. 6 .

numerical approximation schemes is always negligible in comparison with the error of the wavelet approximation. We consider the function

$$
f(x)=\exp \left(-20(x-1 / 2)^{2}\right),
$$

and calculate $\mathscr{P}_{n} f(x)$ for $x \in[0,1]$.

Figure 5 shows the error $\mathscr{E}_{5} f(x)$ in case the scaling function is the orthogonal Daubechies function with $N=2$. The dotted lines are the envelopes of the leading term of the error expansion. At this level the leading term already provides a reasonable approximation of the error. Note that the interpolation properties described in Sect. 7 hold.

Table 2. Error in the Daubechies and spline case $(N=4)$

$\begin{array}{lll}\text { level } & \text { Daubechies } & \text { spline } \\ 1 & 7.53 \mathrm{e}-01 & 3.05 \mathrm{e}-01 \\ 2 & 4.01 \mathrm{e}-01 & 5.07 \mathrm{e}-02 \\ 3 & 5.00 \mathrm{e}-02 & 7.53 \mathrm{e}-03 \\ 4 & 3.16 \mathrm{e}-03 & 3.13 \mathrm{e}-04 \\ 5 & 1.52 \mathrm{e}-04 & 1.01 \mathrm{e}-05 \\ 6 & 9.45 \mathrm{e}-06 & 4.24 \mathrm{e}-07 \\ 7 & 6.06 \mathrm{e}-07 & 2.37 \mathrm{e}-08 \\ 8 & 3.82 \mathrm{e}-08 & 1.53 \mathrm{e}-09 \\ 9 & 2.39 \mathrm{e}-09 & 9.67 \mathrm{e}-11 \\ 10 & 1.50 \mathrm{e}-10 & 6.06 \mathrm{e}-12\end{array}$

Figure 6 shows the error $\mathscr{C}_{5} f(x)$ in case the scaling function is the B-spline of order $N=2$ and the dual scaling function is the one with $\widetilde{N}=2$ constructed in [7]. Again the dotted line are the envelopes of the leading term of the error expansion. One can clearly distinguish the shape of the Bernoulli spline of degree 2.

Table 2 compares $\max _{x}\left|\mathscr{E}_{n} f(x)\right|$ on different levels in two cases. In the first one the scaling function is the orthogonal Daubechies scaling function with $N=4$. The 
second one corresponds to the biorthogonal case with $\varphi(x)=N_{4}(x)(N=4)$ and $\widetilde{N}=6$. The order of convergence is $O\left(h^{4}\right)$ in both cases. On the finer levels the error is indeed divided by 16 each time. This confirms what was predicted in Sect. 8: The approximation using splines at a certain level yields roughly the same error as an approximation using Daubechies' scaling functions with the same $N$ at the next (finer) level.

Acknowledgement. The first author would like to thank Charles Chui and Ewald Quak for interesting discussions on spline wavelets during his visit to Texas A\&M University; Carl de Boor, Ron DeVore, and Sherman Riemenschneider for pointing out valuable references to Euler and Bernoulli splines; and Gilbert Strang for stimulating comments. It was Charles Chui who first remarked that there might be a connection between the $\sigma_{0}(x)$ function and the Euler splines. We also would like to thank Kathy Borg-Todd for helping us with the presentation.

\section{References}

1. Abramowitz, M., Stegun, I.A. (1965): Handbook of mathematical functions. Dover Publications, New York

2. Battle, G. (1987): A block spin construction of ondelettes. Comm. Math. Phys. 110, 601-615

3. De Boor, C. (1976): On the cardinal spline interpolant to $\mathrm{e}^{\mathrm{i} u t}$. SIAM J. Matrix Anal. Appl. 7, 930-941

4. Chui, C.K. (1992): An Introduction to Wavelets. Academic Press, San Diego

5. Chui, C.K., Wang, J.Z. (1991): A cardinal spline approach to wavelets. Proc. AMS 113, 785-793

6. Chui, C.K., Wang, J.Z. (1992): On compactly supported spline wavelets and a duality principle. Trans. AMS 330, 903-915

7. Cohen, A., Daubechies, I., Feauveau, J. (1992): Bi-orthogonal bases of compactly supported wavelets. Comm. Pure Appl. Math. 45, 485-560

8. Daubechies, I. (1988): Orthonormal bases of compactly supported wavelets. Comm. Pure Appl. Math. 41, 909-996

9. Daubechies, I. (1992): Ten Lectures on Wavelets. Number 61 in CBMS-NSF Series in Applied Mathematics. SIAM, Philadelphia

10. Daubechies, I. (1993): Orthonormal bases of compactly supported wavelets II. Variations on a theme. SIAM J. Math. Anal. 24, 499-519

11. Deslauriers, G., Dubuc, S. (1987): Interpolation dyadique. In: Fractals, Dimensions non entières et applications, pp. 44-55, Masson, Paris

12. Deslauriers, G., Dubuc, S. (1989): Symmetric iterative interpolation processes. Constr. Approx. 5, 49-68

13. Donoho, D.L. (1992): Interpolating wavelet transforms. Preprint Department of Statistics, Stanford University

14. Erdélyri, A., Magnus, W., Oberhettinger, F., Tricomi, F.G. (1953): Higher Trancendental Functions, volume I. McGraw-Hill Book Company

15. Fix, G., Strang, G. (1969): Fourier analysis of the finite element method in Ritz-Galerkin theory. Stud. Appl. Math 48, 265-273

16. Janssen, A.J.E.M.: The Zak transform and sampling theorems for wavelet subspaces. Preprint Philips Research Laboratories, Eindhoven, The Netherlands.

17. Janssen, A.J.E.M. (1988): The Zak transform: a signal transform for sampled time-continuous signals. Philips J. Res. 43, 23-69

18. Jawerth, B., Sweldens, W.: An overview of wavelet based multiresolution analyses. SIAM Rev. (to appear)

19. Jia, R.-Q., Micchelli, C.A. (1991): Using the refinement equations for the construction of pre-wavelets II: Powers of two. In: Laurent, P.J, Le Méhauté, A., Schumaker, L.L., eds., Curves and Surfaces. Academic Press, New York

20. Keinert, F. (1994): Biorthogonal wavelets for fast matrix computations. Appl. Comput. Harmon. Anal. 1, (to appear) 
21. Korneichuk, N. (1991): Exact Constants in Approximation Theory. Number 38 in Encyclopedia of mathematics and its applications. Cambridge University Press

22. Lemarié, P.-G. (1988): Ondelettes a localisation exponentielle. J. Math. Pures Appl. 67, 227-236

23. Mallat, S.G. (1989): Multifrequency channel decompositions of images and wavelet models. IEEE Trans. Acoust. Speech Signal Process. 37, 2091-2110

24. Meyer, Y. (1990): Ondelettes et Opérateurs, I: Ondelettes, II: Opérateurs de Calderón-Zygmund, III: (with Coifman, R.), Opérateurs multilinéaires. Hermann, Paris, 1990. English translation of first volume (Wavelets and Operators) is published by Cambridge University Press.

25. Micchelli, C.A. (1991): Using the refinement equations for the construction of pre-wavelets. Numerical Algorithms 1, 75-116

26. Micchelli, C.A, Rabut, C., Utretas, F.I. (1991): Using the refinement equations for the construction of pre-wavelets III: Elliptic splines. Numerical Algorithms 1, 331-352

27. Saito, N., Beylkin, G.: Multiresolution representations using the autocorrelation functions of compactly supported wavelets. Preprint University of Colorado at Boulder.

28. Schoenberg, I.J. (1973): Cardinal Spline Interpolation. Number 12 in CBMS-NSF Series in Applied Mathematics. SIAM Publications, Philadelphia

29. Strang, G. (1989): Wavelets and dilation equations: A brief introduction. SIAM Rev. 31, 614-627

30. Strang, G., Fix, G. (1973): An analysis of the finite element method. Prentice Hall

31. Strang, G., Fix, G. (1973): A Fourier analysis of the finite element variational method. In: Constructive aspects of Functional Analysis. Edizione Cremonese, Rome

32. Sweldens, W.: Asymptotic error expansions for spline wavelets and connections with Euler and Bernoulli splines. Manuscript.

33. Sweldens, W., Piessens, R.: Quadrature formulae and asymptotic error expansions for wavelet approximations of smooth functions. SIAM J. Numer. Anal., (to appear).

This article was processed by the author using the $\mathrm{LAT}_{\mathrm{E}} \mathrm{X}$ style file pljourl from Springer-Verlag. 